\title{
Impulse conduction in multiple sclerosis: a theoretical basis for modification by temperature and pharmacological agents
}

\author{
C. L. SCHAUF AND FLOYD A. DAVIS \\ From the Departments of Neurological Sciences and Biomedical Engineering, Rush Medical College, \\ Rush-Presbyterian-St. Luke's Medical Center, Chicago, Illinois 60612, U.S.A.
}

SYNOPSIS The simplest model for explaining conduction defects in multiple sclerosis (MS) and other demyelinating diseases assumes that the only abnormality present is loss of myelin. The consequences of such an assumption have been investigated by numerical solution of a well-known set of differential equations describing conduction in a model demyelinated axon. In agreement with clinical findings, we show that this model predicts that the temperature at which conduction block occurs is a steep function of the extent of demyelination, so that small temperature increases may block large numbers of conducting fibres. Decreasing calcium concentration (or increasing $\mathrm{pH}$ ) iș calculated markedly to improve the conduction velocity of conducting demyelinated fibres and will $\frac{\mathrm{O}}{\mathrm{D}} \mathrm{N}$ in addition, restore conduction in blocked fibres. The effects of other pharmacological agents have्巳 also been computed. The presence of a demyelinating lesion in a nerve fibre is shown greatly to impai尺 $\vec{D}$ the ability of the fibre to conduct repetitive impulses, conduction failing at much lower frequencies $\bar{D}$ than in normal fibres. These calculations provide some insight into the nature of conduction defect\$ in demyelinated nerve, demonstrate that many clinical features of MS are the expected consequence of loss of myelin and do not require the presence of other defects for their explanation, and provide a useful approach to the search for a symptomatic therapy.

Multiple sclerosis (MS) is a disease of the central nervous system in which the characteristic pathological alteration consists of myelin loss with a relative preservation of axis cylinders. It is generally believed that the clinical signs and symptoms in MS are the result of defects of impulse conduction in demyelinated axons of the central nervous system. A striking example of this is the central visual field defect associated with optic nerve lesions. Here there can be little doubt that the observed scotoma is related to a disturbance of conduction in a specific bundle of topographically oriented demyelinated fibres.

A spectrum of physiological abnormalities is seen in MS patients. In addition to apparent slowing and block of conduction (Namerow, 1968a), some fibres are unable faithfully to conduct repetitive impulses and appear to fatigue rapidly (Namerow, 1972). There is also a curious lability whereby clinical signs and symptoms can fluctuate dramatically with small changes in the internal environment. Thus small changes in body temperature produce dramatic reversible alterations in the neurological signs and symptoms of MS, with heat causing a worsening (Nelson and McDowell, 1959; Namerow, 1968b) and cooling an improvement (Watson, 1959). Experimental evidence suggests that this phenomenon is caused by an effect of temperature on conduction in demyelinated axons (Davis, 1970; Davis and Jacobson, 1971; Rasminsky, 1972). Also, acute transient improvement in scotomas, nystagmus, and oculomotor paresis has been demonstrated in MS patients after procedures known to lower serum calcium concentration (Davis et al., 1970).

The simplest possible model for MS (and presumably for other demyelinating diseases) assumes that the only defect present is loss of myelin without any concomitant pathological 
alterations in the nerve membrane itself. Rasminsky and Sears (1972) have provided strong experimental evidence in rat ventral nerve root fibres demyelinated by injection of diphtheria toxin that the axon membrane in the internode is not excitable. Therefore, previous mathematical models demonstrated to describe conduction in myelinated nerve (Fitzhugh, 1962; Goldman and Albus, 1968) are modified to simulate myelin loss by only altering the myelin resistance and capacitance.

Smith and Koles (1970) and Koles and Rasminsky (1972) have investigated the effects of several morphological patterns of myelin loss (uniform demyelination of one or more internodes and paranodal demyelination) on conduction velocity using such a model system. In all cases they found conduction velocity to decrease with increasing demyelination until finally conduction block occurs, though the amount of myelin just sufficient to sustain conduction depends on the exact morphology.

We have extended calculations based on this model system to attempt to account for the complex clinical phenomena described earlier. These studies provide a unified account of temperature and calcium effects and suggest one possible cause of observed impairment in repetitive impulse conduction. In addition, they indicate a sound basis for the possible development of a symptomatic therapy for demyelinating diseases.

\section{METHODS}

The system of equations describing the model system is identical with that first presented by Goldman and Albus (1968) and modified to account for demyelination by Koles and Rasminsky (1972), except for changes in the voltage-dependent rate constants and permeability constants (equations 9-16 of Goldman and Albus, 1968) to account for the effects of changes in temperature, calcium concentration, and $\mathrm{pH}$, and the application of pharmacological agents.

The model nerve consisted of 20 nodes with stimulating current applied to node 1 and with node 20 shorted to ground. The geometry was identical with that of Goldman and Albus (1968), with normal external myelin diameter chosen to be $30 \mu \mathrm{m}$. Paranodal demyelination is not a characteristic feature of MS, and hence, to account for demyelination, myelin resistance was usually uniformly decreased and myelin capacitance increased by an amount appropriate for the degree of myelin loss (equations 2 and 3 of Goldman and Albus, 1968) and expressed as a fraction of the normal resistance. We assumed demyelination to begin at node 4 and proceed to the end of the fibre, except in those cases where a single demyelinated internode was studied. All constants and initial conditions are given in Goldman and Albus (1968, Appendix). Note that the experimental data from which these equations were derived were obtained at $20^{\circ} \mathrm{C}$, which is consequently the reference temperature for our calculations.

The value of $30 \mu \mathrm{m}$ chosen for normal external myelin diameter is at the upper range of real fibre diameters. This was used in order to have values for conduction velocities which were large, so as to minimize running time for the computer programmes. The work of Goldman and Albus (1968) shows that as long as the relationships between normal external myelin diameter, axis cylinder diameter, and internodal distance remain constant, the only effect of changing fibre diameter is to scale the conduction velocity by a constant factor proportional to diameter.

\section{TABLE}

EXPERIMENTAL EFFECTS OF ALTERATIONS IN TEMPERATURE AND CALCIUM CONCENTRATION

\begin{tabular}{ccc}
\hline Parameter & $Q_{10}{ }^{*}$ & $\begin{array}{c}m \text { V shift per } \dagger \\
\text { e-fold change } \\
\text { in }\left[\mathrm{Ca}^{+}+\right]\end{array}$ \\
\hline$\overline{\mathbf{P}}_{\mathrm{Na}}$ & 1.3 & - \\
$\mathbf{P}_{\mathrm{K}}^{\prime}$ & 1.2 & - \\
$\overline{\mathbf{P}}_{\mathrm{P}}$ & 1.2 & - \\
$\alpha_{\mathrm{m}}$ & 1.8 & 8.6 \\
$\beta_{\mathrm{m}}$ & 1.7 & 8.6 \\
$\alpha_{\mathrm{h}}$ & 2.8 & 7.4 \\
$\beta_{\mathrm{h}}$ & 2.9 & 7.4 \\
$\alpha_{\mathrm{n}}$ & 3.2 & 0.8 \\
$\beta_{\mathrm{n}}$ & 2.8 & 0.8 \\
$\alpha_{\mathrm{p}}$ & $3.0 \ddagger$ & $0.0 \ddagger$ \\
$\beta_{\mathrm{p}}$ & $3.0 \ddagger$ & $0.0 \ddagger$ \\
\hline
\end{tabular}

\footnotetext{
* Data from Frankenhaeuser (1963).

$\dagger$ Data from Hille (1968). Note that Hille presented his results as shifts in time constants and -steady-state levels for the various parameters, not directly as rate constants. The values here are derived from his data.

$\ddagger$ Values assumed. Experimental data not available.
}

The effects of temperature variation were allowed for by scaling all rate constants and permeability constants by the experimentally determined $Q_{10}$ 's (Frankenhaeuser and Moore, 1963; Frankenhaueser, 1965) (Table). This method of incorporating the effect of temperature is justified by the experimental 
data and has been shown to produce in model nerve fibres calculated responses which are in agreement with observations on normal living nerve (Hodgkin and Huxley, 1952; Huxley, 1959; Frankenhaeuser, 1965; Cooley and Dodge, 1966; Smith and Koles, 1970). The effect of temperature on the myelin resistance and capacitance and axoplasmic resistivity is not likely to be a significant factor, but in any case could not be taken into account for lack of adequate experimental data.

The effects of calcium and $\mathrm{pH}$ were calculated by translating the functions describing the voltagedependent rate constants along the voltage axis according to experimental data (Hille, 1968-see Table). This general procedure has also been justified by computations of the effect of calcium on conduction in normal, unmyelinated nerve (Huxley, 1959).

The differential equations were converted to difference form and integrated using a fourth-order Runge-Kutta procedure on IBM $370 / 155$ and SPC

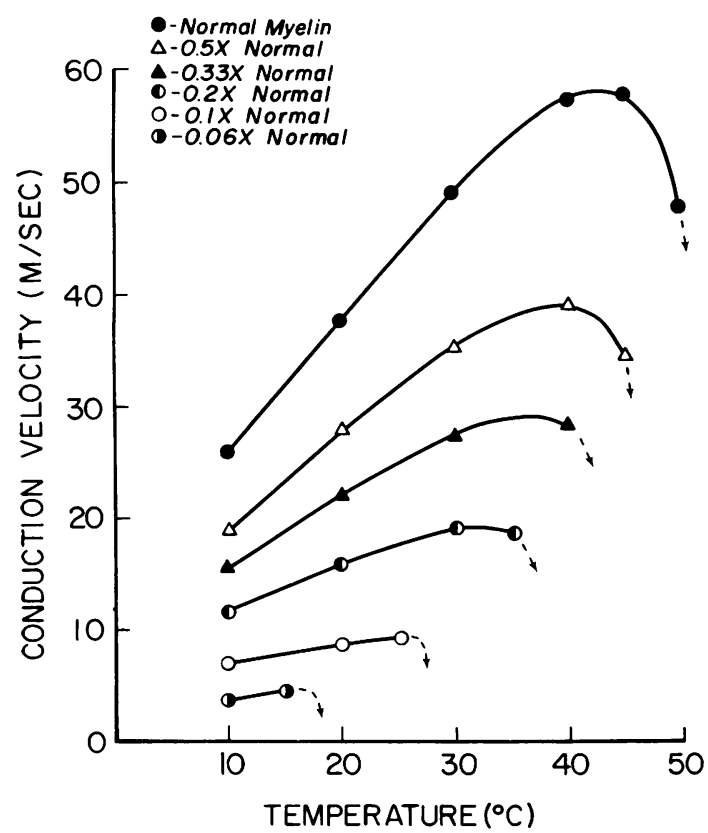

FIG. 1. Calculated values of conduction velocity as a function of temperature for a normal nerve fibre (upper curve) and fibres with increasing loss of myelin. For each curve the last datum point at the right is for the highest temperature at which continuous conduction is possible, the dashed lines and arrows simply being a convenient means of indicating block of conduction at a temperature $3^{\circ} \mathrm{C}$ greater. The amount of demyelination is expressed in terms of the myelin resistance relative to normal.
$16 / 65$ digital computers. In most cases the fibre was sampled at each node and at space increments of $1 / 10$ the internodal distance. Time increments were automatically adjusted to maintain errors in voltage at less than $10 \mu \mathrm{V}$.

\section{NUMERICAL COMPUTATIONS}

EFFECTS OF TEMPERATURE Figures 1 and 2 show the results of the computations we have carried out to examine the combined effects of temperature and loss of myelin on conduction. Figure 1 gives the calculated conduction velocity as a function of temperature for a normal nerve fibre (top curve) and a series of fibres subjected to increasing amounts of demyelination $(0.5,0.33$, $0.2,0.1$, and 0.06 times the normal myelin resistance). In each curve the last point at the right is at the highest temperature at which conduction occurs. A temperature $3^{\circ} \mathrm{C}$ higher does not permit conduction, as indicated by the dashed lines.

The conduction velocity of normal nerve in creases with increasing temperature over a broad? 음 range, reaches a maximum at about $42^{\circ} \mathrm{C}$, and then decreases before block. This result, includ 9

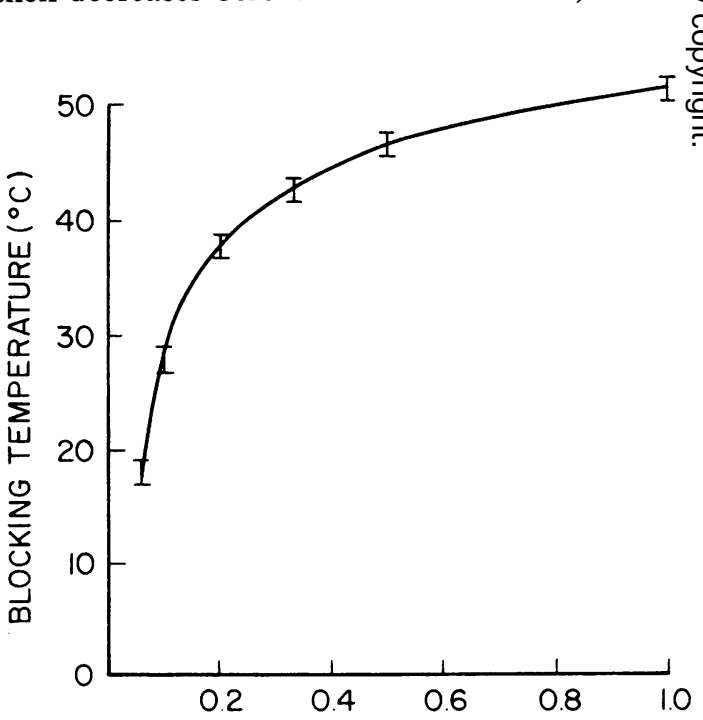

$$
\begin{aligned}
& \text { RELATIVE DEGREE OF MYELINATION } \\
& \text { AS FRACTION OF NORMAL }
\end{aligned}
$$

FIG. 2. Blocking temperature as a function of the amount of myelin loss. The error bars correspond to an uncertainty of $2^{\circ} C$. The amount of demyelination is expressed in terms of the myelin resistance relative to normal. 
ing the calculated presence of a maximum, is similar to that calculated for the squid giant axon (Huxley, 1959). In demyelinated fibres the conduction velocity also generally increases with increasing temperature, although the slope is reduced. In addition, the presence of a maximum and the subsequent decrease become less apparent with increasing loss of myelin.

As myelin is lost, conduction fails at progressively lower temperatures. Particularly with substantial amounts of demyelination, the blocking temperature is a steep function of the amount of myelin present (Fig. 2). To put the result another way, given a population of fibres with varying amounts of myelin lost (such as would be expected to occur in a real lesion), a small increase in temperature would substantially decrease the number of fibres whose blocking temperature exceeds the body temperature and thus will reduce the number of conducting fibres. At the same time that more fibres are blocked, the conduction velocity of those fibres which remain conducting will generally tend to be increased.

Since a demyelinated axon is seen to be in a delicate balance between conducting and blocked states, any factor serving to depress function slightly would have dramatic effects on demyelinated nerve, although possibly having little noticeable effect on normal fibres. Thus, decreasing the maximum sodium permeability by $20 \%$ decreases the action potential in normal nerve by only $3 \mathrm{mV}$, yet we calculate it to cause a further $5^{\circ} \mathrm{C}$ lowering of the blocking temperature of a $90 \%$ demyelinated fibre.

Decreases in axis cylinder diameter (Greenfield, 1958), due possibly to oedema, can occur in multiple sclerosis. The lower curve in Fig. 6 shows the effect of a $50 \%$ decrease in diameter occurring in a demyelinated region of nerve. The blocking temperature is decreased (middle curve) by an additional $15^{\circ} \mathrm{C}$ for any particular amount of demyelination. Obviously, a substantial degree of additional clinical dysfunction would be expected to result from such a situation.

EFFECTS OF CALCIUM AND pH Figure $3 \mathrm{a}$ illustrates the effect of an e-fold (approximately $63 \%$ ) decrease in calcium concentration on conduction in fibres with $10 \%$ and $20 \%$ normal myelin. The solid curves are reproduced from Fig. 1 for

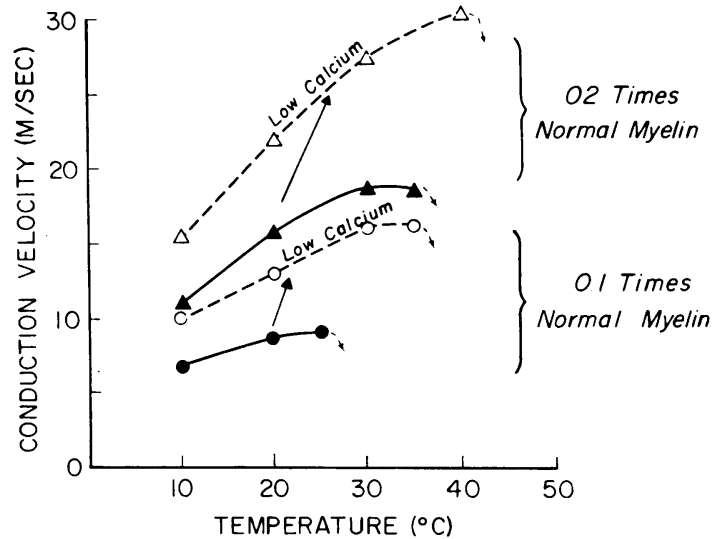

FIG. 3a. Conduction velocity as a function of temperature calculated for fibres with $10 \%$ normal myelin resistance $(O, O)$ and $20 \%$ normal $(\triangle, \Delta)$ in normal Ringer's solution (O, $\Delta$-from Fig. 3) and Ringer's solution with $1 / e^{\text {th }}$ normal calcium $(\bigcirc, \triangle)$. As in Fig. 3, the datum point at the highest temperature in each curve represents the maximum temperature at which continuous conduction is possible.

convenience. As in Fig. 1, the last point on the right is at the highest temperature capable of sustaining conduction. In both cases, at temperatures lower than the blocking temperature, a decrease in calcium concentration increases conduction velocity by $40-50 \%$. In addition to this, reductions in calcium concentration cause the

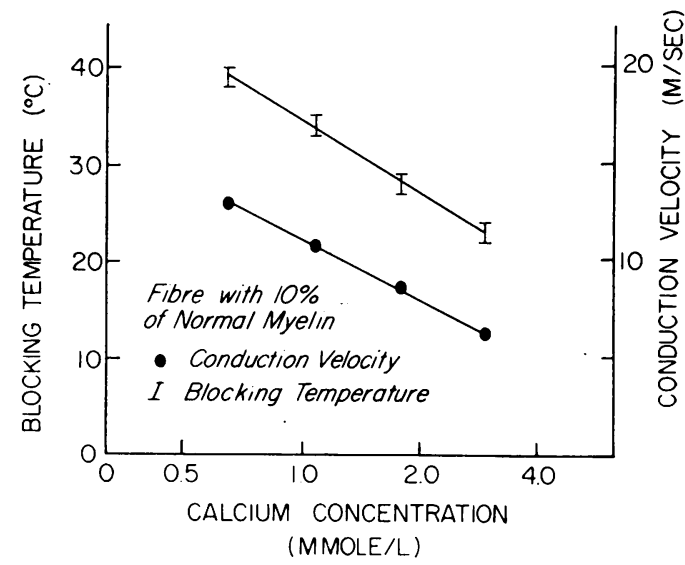

FIG. 3b. Blocking temperature (error bars) and conduction velocity (O) as a function of the calcium concentration for a fibre with $10 \%$ normal myelin resistance. Note that the abscissa is logarithmic. 
blocking temperature to increase toward normal, and this effect is most predominant in severely demyelinated fibres.

Figure $3 b$ shows that both the blocking temperature and the conduction velocity are linear functions of the logarithm of the calcium concentration. This figure is for a fibre with $10 \%$ normal myelination. If a larger amount of myelin is present, the slope of the curve relating blocking temperature to calcium concentration is decreased.

Thus, the result of decreasing the calcium concentration in a population of fibres having different amounts of myelin lost is predicted to be an increase in the number of conducting fibres, combined with a restoration of the conduction velocity toward more normal values in those fibres which were not blocked at normal calcium levels. This is in contrast with the effects of temperature in which cooling increases the number of conducting fibres but decreases conduction velocity in those which were not blocked.

Increasing $\mathrm{pH}$ acts on the rate constants in the same direction as decreases in calcium concentration. Detailed calculations were not performed, but since the rate constants shift by only $1.3 \mathrm{mV}$ per e-fold change in hydrogen ion concentration $(3.0 \mathrm{mV}$ per $\mathrm{pH}$ unit) at physiological $\mathrm{pH}$ (Hille, 1968), we may conclude that this alteration would be much less effective than similar changes in calcium concentration.

Effects qualitatively similar to these have also been calculated for the other morphological patterns of myelin loss considered by Koles and Rasminsky (1972). Reducing calcium concentra- tion will restore conduction in a fibre blocked by demyelination of a single internode or by paranodal demyelination.

RESPONSES TO REPETITIVE STIMULATION The response of the model axon to a second stimulus delivered at a variable time after an initial stimulus is shown in Fig. 4 for both a normal nerve and a nerve which has lost all but $10 \%$ of its myelin. The action potential amplitude and conduction velocity resulting from the initial stimulus were $110.4 \mathrm{mV}$ and $37.8 \mathrm{~m} / \mathrm{sec}$ for normal nerve, and $90.0 \mathrm{mV}$ and $8.7 \mathrm{~m} / \mathrm{sec}$ for the $90 \%$ demyelinated fibre. To prepare Fig. 4 , the magnitude and conduction velocity of the response produced by a second impulse were divided by the corresponding values for the initial response for both normal and demyelinated nerve.

For normal nerve, a second stimulus at 1.85 msec after the initial stimulus is unable to produce a response, whereas at $2.04 \mathrm{msec}$ a second $\mathrm{O}$ response with initial amplitude and conduction $\cong$ velocity of $69 \mathrm{mV}$ and $14.6 \mathrm{~m} / \mathrm{sec}$ is produced. $\stackrel{\mathbb{}}{-}$ Intermediate times were not explored. With in creasing time between the stimuli the amplitude $ᄋ$ 을 and velocity of the second response increase rapidly toward initial values. During the intervato in which a second response is reduced, the second? response is continually lagging behind the initial impulse, while gradually recovering its amplitude and velocity. Whether substantial changes in frequency occur depends on the conduction distance involved.

In the case of a $90 \%$ demyelinated fibre, a

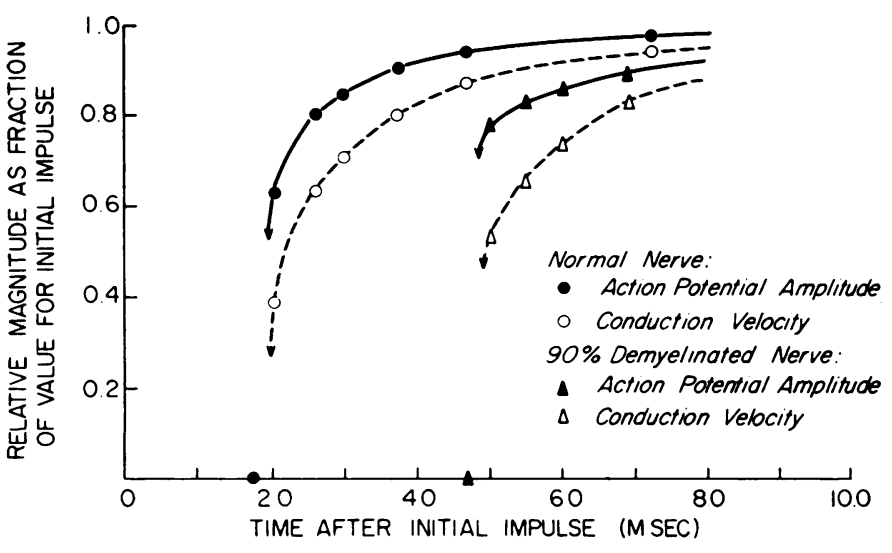

FIG. 4. Calculated responses of a normal nerve and a nerve with $10 \%$ normal myelin resistance to pairs of stimuli. The abscissa is the time between stimuli. The ordinate plots the ratio action potential amplitude elicited by second stimulus $\overline{\text { action potential amplitude elicited }}$ by initial stimulus

and a similar ratio for the conduction velocities. The temperature is $20^{\circ} \mathrm{C}$. At intervals less than 1.85 msec (normal nerve) and 4.7 msec (demyelinated nerve) a second response cannot be elicited. These points are indicated on the abscissa. 


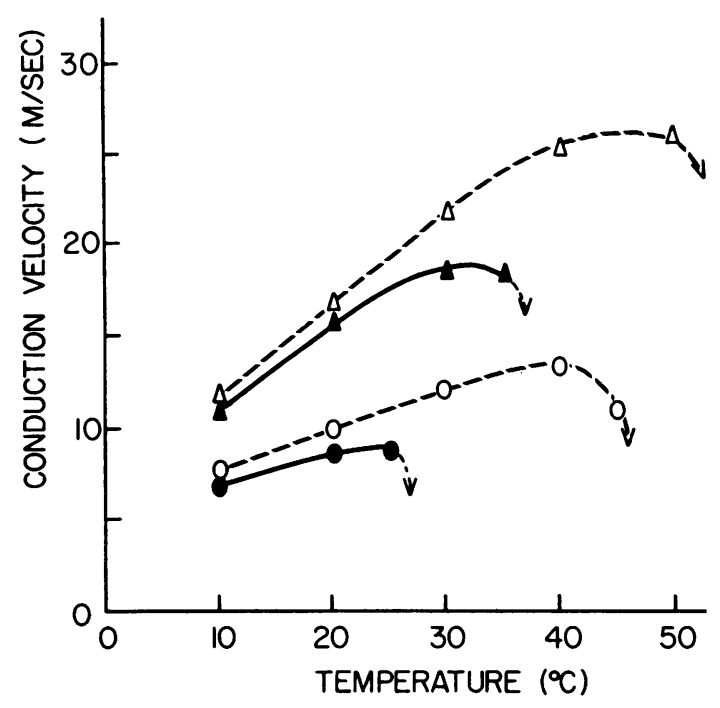

FIG. 5. Conduction velocity as a function of temperature calculated for fibres with $10 \%$ normal myelin resistance $(O, O)$ and $20 \%$ normal $(\triangle, \Delta)$ for the situation in which the inactivation time constant is normal (O, $\mathbf{\Delta}$-from Fig. 3) and the case where the inactivation time constant is increased by a factor of 4 $(\bigcirc, \triangle)$.

second stimulus at $4.7 \mathrm{msec}$ fails to produce a response, and at $5.0 \mathrm{msec}$ a response with initial amplitude of $70 \mathrm{mV}$ and velocity of $4.7 \mathrm{~m} / \mathrm{sec}$ results. Again, intermediate times were not examined. With an interval of more than $5 \cdot 0$ msec, the initial amplitude and velocity again recover.

Therefore, if we consider a pair of impulses $4.7 \mathrm{msec}$ apart travelling in a fibre which contains a zone which is $90 \%$ demyelinated, only the first impulse will pass through. With larger amounts of myelin loss, the situation rapidly becomes worse.

This increase in refractory period of transmission (RPT) from $1.95 \pm 0.1 \mathrm{msec}$ in normal nerve to $4.85 \pm 0.15 \mathrm{msec}$ in $90 \%$ demyelinated nerve is much larger than that which would be expected simply from a consideration of the time course of the permeability variables during the action potential. In $90 \%$ demyelinated nerve at $4.0 \mathrm{msec}$ after the start of the initial response, the values of the permeability variables have recovered to values which are as close or closer to their resting states than those at $3.2 \mathrm{msec}$ in normal nerve, where conduction is nearly normal. The fibre remains blocked because of the much greater shunting of current in the demyelinated internode.

Such increases in refractory period of transmission are most noticeable in fibres demyelinated nearly to the point of conduction block. Thus, a fibre with $20 \%$ normal myelin resistance is calculated to have an RPT of $2.65 \pm 0.1 \mathrm{msec}$, which is an increase of only $35 \%$ relative to normal, compared with the $250 \%$ increase seen in the case of $90 \%$ demyelination. A fibre with $50 \%$ normal myelin resistance shows an almost normal RPT of $2 \cdot 1 \pm 0 \cdot 1 \mathrm{msec}$.

Decreases in calcium concentration are calculated to improve substantially the ability of demyelinated nerve to transmit repetitive impulses. Thus, for $90 \%$ demyelinated nerve, an e-fold decrease in calcium concentration results in a decrease in the refractory period of transmission from $4.85 \pm 0.15 \mathrm{msec}$ to $3.6 \pm 0.1 \mathrm{msec}$. Larger effects of calcium are observed in fibres which are more severely demyelinated.

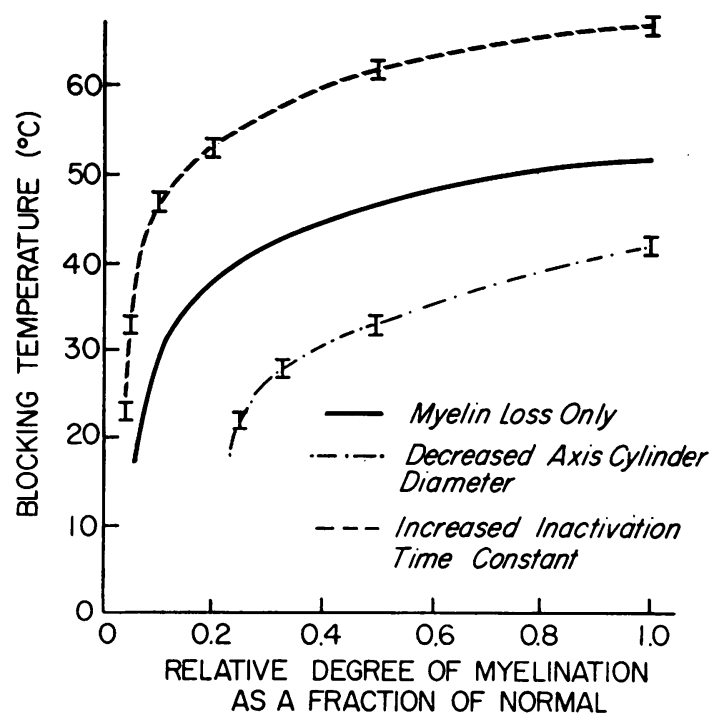

FIG. 6. Blocking temperature as a function of the relative myelin resistance for the situation in which the inactivation time constant has been increased by $a$ factor of 4 (-_-) and for the case where in addition to loss of myelin there is a twofold decrease in axis cylinder diameter (-.---). The curve of Fig. 2 for myelin loss alone is reproduced for convenience. 
PHARMACOLOGICAL EFFECTS This model system provides a convenient means of defining the manner in which the kinetic properties of the nerve membrane should be altered in order to improve conduction in demyelinated nerve. Presumably such effects occurring in MS patients would improve clinical signs and symptoms. We have already described the effects of temperature, calcium, and $\mathrm{pH}$. Conduction in demyelinated nerve is improved by factors which increase the duration of the action potential without adversely affecting conduction velocity or action potential amplitude, since the total membrane current is increased. This may be accomplished by reducing the contribution of the steady-state conductance, or prolonging the sodium conductance by slowing the inactivation process.

Figures 5 and 6 illustrate the effect of a fourfold increase in the sodium inactivation time constant, $\tau_{\mathrm{h}}$. This alteration results in an action potential in normal nerve which is about 2.5 times as long. Figure 5 shows that when applied to a demyelinated fibre, this procedure does not appreciably affect the conduction velocity but greatly increases the blocking temperature for any amount of demyelination. This is more clearly seen in the upper curve of Fig. 6. The curve relating blocking temperature and amount of myelin is shifted vertically by more than $15^{\circ} \mathrm{C}$. Thus at any given temperature conduction is possible with far less myelin.

Reduction of the maximum potassium permeability will also improve conduction in demyelinated nerve by increasing the blocking temperature, but the effects are not as dramatic. Thus, a $50 \%$ decrease in $\mathrm{P}^{\prime}{ }_{\mathrm{k}}$ increases the blocking temperature of an $80 \%$ demyelinated fibre by only $2^{\circ} \mathrm{C}$. This is due to the fact that the increasing potassium permeability does not contribute as much to the falling phase of the action potential as the simultaneous inactivation of the sodium permeability. A $50 \%$ decrease in maximum potassium permeability prolongs the normal action potential only by about $10 \%$ and does not affect conduction velocity at all.

\section{DISCUSSION}

It should be stressed that the procedure of using a mathematical model to study the behaviour of a demyelinated axon has a great deal of experi- mental justification. Fitzhugh (1962) first described the procedure for combining the phenomenological description of the behaviour of excitable membranes contained in the Hodgkin-Huxley equations (Hodgkin and Huxley, 1952) with the equations for a passive cable (Hodgkin and Rushton, 1946) to yield a description of conducted action potentials in myelinated nerve. Goldman and Albus (1968) extended this work by replacing the Hodgkin-Huxley equations, which describe the behaviour of the squid giant axon, with a similar set of relationships (the Frankenhaeuser-Huxley equations) which were developed by the application of the voltage clamp technique to the myelinated nerve fibre (Dodge and Frankenhaeuser, 1958, 1959; Frankenhaeuser, 1959, 1960, 1962a-c, 1963a, b; Frankenhaeuser and Huxley, 1964).

The calculations of these and other investigators concerning the behaviour of normal myelinated fibres (Frankenhaeuser and Huxley, 1964; Frankenhaeuser, 1965; Hutchinson et al., 1970), combined with similar computations of membrane action potentials (Huxley, 1959 Fitzhugh, 1960; Fishman, 1970) and propagated activity (Cooley and Dodge, 1966) in nonmyelinated fibres, have provided compelling evidence for accepting these models as excellento descriptions of living nerve fibres under a variety of environmental conditions.

The calculations of Smith and Koles (1970) and Koles and Rasminsky (1972), when compared with the experimental data of Rasminsky and Sears (1972), demonstrate the ability of this model to simulate accurately the behaviour of real demyelinated fibres.

Koles and Rasminsky (1972 briefly reported one calculation concerning the increased velocity and subsequent block produced by warming a paranodally demyelinated fibre barely conducting at $20^{\circ} \mathrm{C}$, and mentioned that this qualitatively agreed with experimental observations of Rasminsky (1971) on rat ventral root fibres demyelinated by diphtheria toxin. The complete family of curves shown in Fig. 1 contains quite complex behaviour, and it would be an excellent test of the model's accuracy to see if there is agreement with experimental observations over the entire range.

The calculated relationships of Figs 1 and 2, in addition to indicating that increased numbers 
of demyelinated fibres are blocked with temperature elevation, suggest that the clinical worsening with hyperthermia seen in MS patients (Nelson and McDowell, 1959; Watson, 1959; Namerow, $1968 \mathrm{~b}$ ) is caused by block of conduction alone, and not by any change in conduction velocity. Since the low conduction velocity in demyelinated nerve increases with increased temperature, this factor would, if anything, be expected to improve clinical function.

We may also offer an explanation for the observation that increasing temperature enlarges the size of a central scotoma. There exists compelling evidence that a plaque enlarges by peripheral extension (Lumsden, 1970), with the severest or completely demyelinated fibres generally at the centre, and lesser degrees of involvement toward the periphery. Since totally demyelinated internodes are already blocked (Fig. 1), the blocking effect of hyperthermia is most likely to be on the partially demyelinated axons distributed in greater numbers more peripherally.

This model provides a reasonable explanation for the effects of hypocalcaemia observed clinically. In addition to the acute improvement in scotomas, nystagmus, and oculomotor paresis with intravenous $\mathrm{NaHCO}_{3}$ or edetate (EDTA) referred to earlier, a more recent study indicates that large doses of oral phosphate cause improvement in visual function measured by acuity, field, and pupillometry tests (Becker et al., 1973). Hypocalcaemia is calculated to restore conduction in blocked demyelinated fibres and to increase substantially the conduction velocity of conducting fibres. Both changes would tend to restore function. Note that $\mathrm{NaHCO}_{3}$ serves to increase $\mathrm{pH}$ in addition to decreasing calcium concentration, and that both alterations are calculated to be beneficial.

In segmentally demyelinated peripheral nerves, conduction failure has been observed at lower frequencies than in normal nerve (Cragg and Thomas, 1964; Davis, 1972). Patients with MS have been observed to exhibit defects in the cortical evoked response after peripheral nerve stimulation at frequencies below $100 \mathrm{~Hz}$ (Namerow, 1972). McDonald and Sears (1970) studied lesions induced in the dorsolateral sulcus of the cat spinal cord by injection of diphtheria toxin and found the refractory period for in situ stimulation to be increased by up to fourfold in fibres traversing the demyelinating lesion. The prediction of this model that the absolute refractory period is increased to approximately 2.5 times normal in a $90 \%$ demyelinated fibre seems in reasonable accord with these observations. The implications of such defects for information processing in the central nervous system are enormous, the presence of a demyelinating lesion causing the fibre to act as a filtering device.

The effects of decreased potassium permeability and increased inactivation time constant were studied because there exist pharmacological agents having primarily these effects. Thus, tetraethylammonium (TEA) chloride will block the potassium permeability of myelinated nerve quite specifically (Hille, 1967). Both DDT and scorpion venom slow the sodium inactivation process, although both are irreversible, tend to be progressive in their effect, and are not completely specific for sodium inactivation (Narahashi and Haas, 1968, 1972). Condylactis toxin very specifically slows sodium inactivation but shares the disadvantage of being irreversible and progressive (Narahashi et al., 1969).

Though these particular agents have not been tested in model demyelinated nerve, the computations do serve to indicate the direction a pharmacological approach should take in terms of the underlying kinetic processes. Agents which serve to decrease the contribution of the potassium permeability are not likely to be very satisfactory in restoring conduction to demyelinated fibres because the time course of the action potential in this model system is much less sensitive to changes in potassium activation than sodium inactivation. For clinical purposes, it is suggested that it might be fruitful to try to develop an agent having a specific, reversible, and easily controlled effect on the sodium inactivation process.

We have attempted to extend a well-known theoretical model of impulse conduction in demyelinated nerve in order to understand better a variety of physiological abnormalities and to point out related clinical implications. In the present instance, the model says in a rather striking way that many of the pathophysiological and clinical features of MS are accounted for on the assumption that myelin loss is the only defect 
present. Also, the theoretical effects of a variety of pharmacological agents on conduction in demyelinated nerve can be predicted given a sufficiently detailed understanding of their effects on normal nerve fibres. Whether this approach will lead to any clinically useful therapeutic agent is unknown, but the basic idea seems scientifically sound and worthy of further investigation.

We thank Dr. T. Hoeppner, Dr. J. A. Michael, and Dr. F. Morrell for their helpful comments about this work, and Mrs. Valerie Meineke for her effort in preparing the manuscript. This investigation was supported by the Morris Multiple Sclerosis Research Fund.

\section{REFERENCES}

Becker, F. O., Michael, J. A., and Davis, F. A. (1973). Acute effect of oral phosphate on visual function in multiple sclerosis. Abstracts of the 1973 Annual Meeting, American Academy of Neurology. Neurology, 23, 431.

Cooley, J. W., and Dodge, F. A., Jr. (1966). Digital computer solutions for excitation and propagation of the nerve impulse. Biophysical Journal, 6, 583-599.

Cragg, B. G., and Thomas, P. K. (1964). Changes in nerve conduction in experimental allergic neuritis. Journal of Neurology, Neurosurgery, and Psychiatry, 27, 106-115.

Davis, F. A. (1970). Axonal conduction studies based on some considerations of temperature effects in multiple sclerosis. Electroencephalography and Clinical Neurophysiology, 28, 281-286.

Davis, F. A. (1972). Impairment of repetitive impulse conduction in experimentally demyelinated and pressureinjured nerves. Journal of Neurology, Neurosurgery, and Psychiatry, 35, 537-544.

Davis, F. A., Becker, F. O., Michael, J. A., and Sorensen, E. (1970). Effect of intravenous sodium bicarbonate, disodium edetate $\left(\mathrm{Na}_{2}\right.$ EDTA), and hyperventilation on visual and oculomotor signs in multiple sclerosis. Journal of Neurology, Neurosurgery, and Psychiatry, 33, 723-732.

Davis, F. A., and Jacobson, S. (1971). Altered thermal sensitivity in injured and demyelinated nerve. Journal of Neurology, Neurosurgery, and Psychiatry, 34, 551-561.

Dodge, F. A., and Frankenhaeuser, B. (1958). Membrane currents in isolated frog nerve fibre under voltage clamp conditions. Journal of Physiology, 143, 76-90.

Dodge, F. A., and Frankenhaeuser, B. (1959). Sodium currents in the myelinated nerve fibre of Xenopus laevis investigated with the voltage clamp technique. Journal of Physiology, 148, 188-200.

Fishman, H. M. (1970). Direct and rapid description of the individual ionic currents of squid axon membrane by ramp potential control. Biophysical Journal, 10, 799-817.

Fitzhugh, R. (1960). Thresholds and plateaus in the HodgkinHuxley nerve equations. Journal of General Physiology, 43, 867-896.

Fitzhugh, R. (1962). Computation of impulse initiation and saltatory conduction in a myelinated nerve fiber. Biophysical Journal, 2, 11-21.

Frankenhaeuser, B. (1959). Steady state inactivation of sodium permeability in myelinated nerve fibres of Xenopus laevis. Journal of Physiology, 148, 671-676.
Frankenhaeuser, B. (1960). Quantitative description of sodium currents in myelinated nerve fibres of Xenopus laevis. Journal of Physiology, 151, 491-501.

Frankenhaeuser, B. (1962a). Delayed currents in myelinated nerve fibres of Xenopus laevis investigated with voltage clamp technique. Journal of Physiology, 160, 40-45.

Frankenhaeuser, B. (1962b). Instantaneous potassium currents in myelinated nerve fibres of Xenopus laevis. Journal of Physiology, 160, 46-53.

Frankenhaeuser, B. (1962c). Potassium permeability in myelinated nerve fibres of Xenopus laevis. Journal of Physiology, 160, 54-61.

Frankenhaeuser, B. (1963a). A quantitative description of potassium currents in myelinated nerve fibres of Xenopus laevis. Journal of Physiology, 169, 424-430.

Frankenhaeuser, B. (1963b). Inactivation of the sodiumcarrying mechanism in myelinated nerve fibres of Xenopus laevis. Journal of Physiology, 169, 445-451.

Frankenhaeuser, B. (1965). Computed action potential in nerve from Xenopus laevis. Journal of Physiology, 180, 780-787.

Frankenhaeuser, B., and Huxley, A. F. (1964). The action potential in the myelinated nerve fibre of Xenopus laevis as computed on the basis of voltage clamp data. Journal of Physiology, 171, 302-315.

Frankenhaeuser, B., and Moore, L. E. (1963). The effect of temperature on the sodium and potassium permeability changes in myelinated nerve fibres of Xenopus laevis. Journal of Physiology, 169, 431-437.

Goldman, L., and Albus, J. S. (1968). Computation of impulse conduction in myelinated fibers: theoretical basis of the velocity-diameter relation. Biophysical Journal, 8, 596607.

Greenfield, J. G. (1958). Demyelinating diseases. In Greenfield's Neuropathology, pp. 441-474. Arnold: London.

Hille, B. (1967). The selective inhibition of delayed potassium currents in nerve by tetraethylammonium ion. Journal of General Physiology, 50, 1287-1302.

Hille, B. (1968). Charges and potentials at the nerve surface. Divalent ions and pH. Journal of General Physiology, 51, 221-236.

Hodgkin, A. L., and Huxley, A. F. (1952). A quantitative description of membrane current and its application to conduction and excitation in nerve. Journal of Physiology, $117,500-544$.

Hodgkin, A. L., and Rushton, W. A. H. (1946). The electrical constants of a crustacean nerve fibre. Proceedings of the Royal Society of London B., 133, 444-479.

Hutchinson, N. A., Koles, Z. J., and Smith, R. S. (1970). Conduction velocity in myelinated nerve fibres of Xenopus laevis. Journal of Physiology, 208, 279-289.

Huxley, A. F. (1959). Ion movements during nerve activity. Annals of the New York Academy of Sciences, 81, 221-246.

Koles, Z. J., and Rasminsky, M. (1972). A computer simulation of conduction in demyelinated nerve fibres. Journal of Physiology, 227, 351-364.

Lumsden, C. E. (1970). The neuropathology of multiple sclerosis. In Handbook of Clinical Neurology, Vol. 9, pp. 217-309. Edited by P. J. Vinken and G. W. Bruyn. NorthHolland: Amsterdam.

McDonald, W. I., and Sears, T. A. (1970). Effect of a demyelinating lesion on conduction in the central nervous system studied in single nerve fibres. Journal of Physiology, 207, 53-54P.

Namerow, N. S. (1968a). Somatosensory evoked responses in multiple sclerosis. Bulletin of the Los Angeles Neurological Society, 33, 74-81. 
Namerow, N. S. (1968b). Circadian temperature rhythm and vision in multiple sclerosis. Neurology (Minneap.), 18, 417422.

Namerow, N. S. (1972). The pathophysiology of multiple sclerosis. In Multiple Sclerosis. Immunology, Virology, and Ultrastructure, pp. 143-181. Edited by F. Wolfgram, G. W. Ellison, J. G. Stevens, and J. M. Andrews. Academic Press: New York.

Narahashi, T., and Hass, H. G. (1968). Interaction of DDT with the components of lobster nerve membrane conductance. Journal of General Physiology, 51, 177-198.

Narahashi, T., Moore, J. W., and Shapiro, B. I. (1969). Condylactis toxin: interaction with nerve membrane ionic conductances. Science, 163, 680-681.

Narahashi, T., Shapiro, B. I., Deguchi, T., Scuka, M., and Wang, C. M. (1972). Effects of scorpion venom on squid axon membranes. American Journal of Physiology, 222 , 850-857.
Nelson, D. A., and McDowell, F. (1959). The effects of induced hyperthermia on patients with multiple sclerosis. Journal of Neurology, Neurosurgery, and Psychiatry, 22, 113-116.

Rasminsky, M. (1972). Internodal Conduction in Normal and Demyelinated Mammalian Nerve Fibres. Ph.D. Thesis, University of London.

Rasminsky, M., and Sears, T. A. (1972). Internodal conduction in undissected demyelinated nerve fibres. Journal of Physiology, 227, 323-350.

Smith, R. S., and Koles, Z. J. (1970). Myelinated nerve fibres: computed effect of myelin thickness on conduction velocity. American Journal of Physiology, 219, 1256-1258.

Watson, C. W. (1959). Effect of lowering of body temperature on the symptoms and signs of multiple sclerosis. New England Journal of Medicine, 261, 1253-1259. 